\title{
Influence of partial immersion on steel plate dynamic proper- ties
}

\author{
Jan Kůrečka ${ }^{1, \star}$ and Vladimír Habán ${ }^{1, \star \star}$ \\ ${ }^{1}$ Viktor Kaplan Department of Fluid Engineering, Brno University of Technology, Technická 2896/2, Brno, 616 69, Czech Republic
}

\begin{abstract}
Eigenfrequency of object drops when it is submerged in the water compared to an air exposition. In this article the effect of partial submersion on dynamic properties of steel plate is investigated. Changes differ by a type of particular modeshape because of relative position of nodes and antinodes to free surface. Dynamic properties are influenced mainly by position of antinodes, in which maximal displacement occurs. Comparison of experiment, FEM simulation in ANSYS and custom code analytical solution is presented. Added effects of fluid on the steel plate are discussed.
\end{abstract}

\section{Introduction}

Change of natural frequency occures when object is submerged in water. Immersion in water causes the drop of eigen frequencies of particular modes to $50-70 \%$ of value in air (sometimes called dry frequency and opposite wet frequency). There is different change of ratio between wet and dry frequency for different modes. Bending (flex) modes are generaly more influenced compared to torsion (twisting) modes which experience smaller frequency drop. Effects on dynamic properties of composite plates were studied in [1] and comparison of free vibration FEM simulation and analytical method is given. Frequencies of different shapes and immersion induced change is presented but only for completly submerged composite plate.

Comparison of computational results with added mass coefficient replacing effects of surruning water is presented in [2]. Analogy of this approach is used in this paper but implemented in a different way as an added density in a transfer matrix method. Added mass effects with a respect to a submersion of a NACA profile in a FEM simulation and experiment wss a subject of study [3]. This contribution is in a way similar, FEM analysis and experiment is compared and an effect of partial submersion on a dynamic properties of studied geometry is presented.

Study [4] focused on a analytical or semi-analytical method to calculate frequency of partially immersed circular cylinder and discussed added mass efect as a defined coefficient. There is presented formula for simple calculation of frequency during partial submersion using this coefficient.

More practical use of added mass effect and a frequency change of solid body is presented in [5] focused

\footnotetext{
^e-mail: 133644@vutbr.cz

$\star \star$ e-mail: haban@fme.vutbr.cz
}

on a marine hydrokinetic turbine and its dynamics influenced by a submersion in a water.

This contribution provides a comparison of a frequency change of steel plate caused by partial immersion in water. Besides experiment the used methods are transfer matrix method (TMM) and FEM analysis in ANSYS software package. Which one of these two methods provides better results compared to experimental measurement is examined.

\section{Experiment}

The experiment consisted of measured steel plate of defined size equiped with accelerometers and either fully submerged (not submerged) or partially while hanging on a steel cables. After excitation the response of sensors was recorded and later transfered to amplitude-frequency space using Fourier transform. The experimental data were obtained as a part of diploma thesis [6].

Dimensions of plate are $550 \mathrm{~mm}(L) 80 \mathrm{~mm}(b)$ and $6 \mathrm{~mm}(t)$, values were chosen so the natural frequencies would be low enough for sensors and frequency change of wetted plate would be big enough to measure and compare to other methods presented in the article. Two sensors were screw mounted in top corners. Geometrical properties are in Fig. 1. Distinction of torsion and bending modes is possible with this assembly, because sensors will have different phase angle in case of a torsion mode and equal for a bending eigen frequency.

The measurement procedure started with excitation of still hanging steel plate with either a hit of a hammer, modal hammer or in case of almost (and fully) submerged plate hammer striking a tool touching a steel plate. This was followed by an arbitrary number of seconds of data gathering during shock damping before next excitation. 


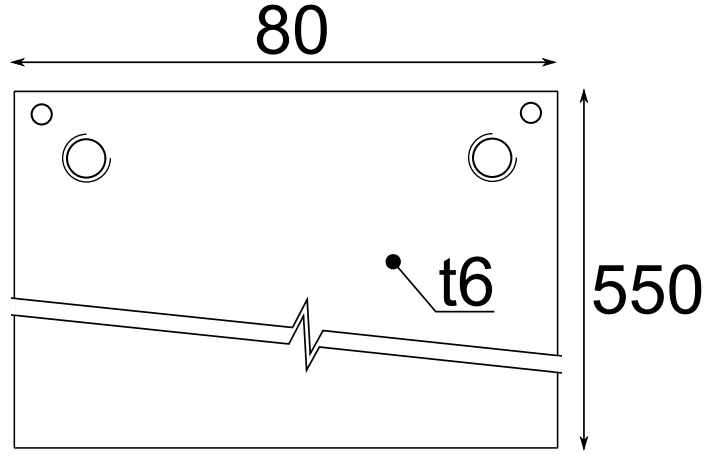

Fig. 1. Dimensions and mounting positions of acceleration sensors

This procedure was repeated with various tools a for different levels of submersion.

Due to equipment limitations the case of almost completly and fully submerged positions of plate could be measured only with one available waterproof sensor. The order of modes and change of order of fifth mode from torsion to bending (dry fifth torsion mode becomes sixth wet frequency) happens before the steel plate is fully submerged. Also the measurement was consistent through repeated events so this limitation was deemed negligible. The results of experimental measurement for dry and wet (fully submerged plate) are in Tab. 1.

Identification of torsion shapemode is rather simple due to mounting position of sensors. In Fig. 1 are mounting positions in top corners of plate and in Fig. 3 is apparent difference for torsion modes. Bending modes are vibrating in a same phase, the phase angle difference is null and torsion modes have phase difference equal to $\pi$ (Pi). This way the change of order of modeshapes could be determined.

In Fig. 2 is experimentaly obtained trend of frequency change for second torsion mode and fourth bending mode during submersion (they are fifth and sixth modes in overall order). This graph indicates that the change happens when there is about $100 \mathrm{~mm}$ of plate still sticking up from the water. Another point to note in this picture is a trend of torsion mode frequency during submersion. With a knowledge of a sixth shape mode in Fig. 3 it is evident that this
Table 1. Experimental results (bold values of wet frequency indicates change between flex and twist mode)

\begin{tabular}{l|l|l} 
Mode & Dry frequency $[\mathrm{Hz}]$ & Wet freq. $[\mathrm{Hz}]$ \\
\hline 1 & 106.1 & 74 \\
2 & 293.5 & 206 \\
3 & 430.6 & 355 \\
4 & 571 & 417 \\
5 & 878 & $\mathbf{7 0 3}(6)$ \\
6 & 948 & $\mathbf{7 2 1}(5)$
\end{tabular}

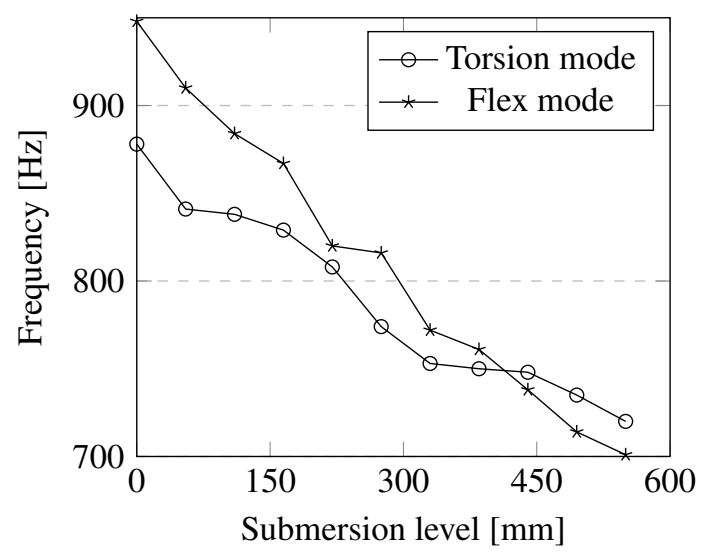

Fig. 2. Second torsion and fourth bending mode during immersion, experimental data

trend is driven by location of nodes and anti-nodes with respect to water level. When the anti-node (location of maximum amplitude of the steel plate movement) is submerged rapid frequency drop follows and vice versa, described torsion mode has a three anti-nodes and two nodes in lenghtwise direction (direction of a submersion) and the line connecting points has a three significant recessive parts. This

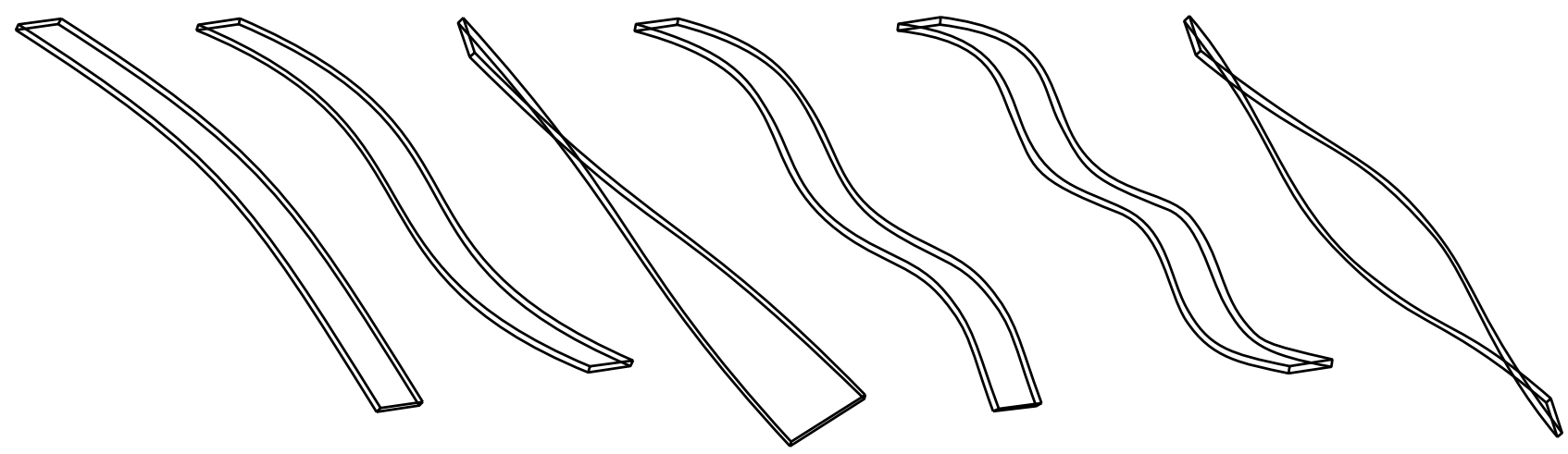

Fig. 3. Shapes of first six eigen modes, third and sixth mode are torsion (twisting) ones rest are bending (flex) modes. Depicted order is valid for sumberged steel plate. 
effect is much less obvious in a frequency-submersion dependency of the compared flex mode because this mode has a six anti-nodes and so the gradual submersion effect is not visible in an eleven step experiment process.

\section{Simulation}

Analogical setup was used to simulate same situation using FEM solver ANSYS with Acoustic extension which makes available acoustic capablities to user in graphic interface. Modal analysis module was used to find eigen frequencies of particular modes. Simulation was split into three parts, simulation of the wet plate with structural and fluid part, simulation of the structural part only (since the effect of air as a surrounding medium is insignificant) and third setup was parametrical case of gradual submersion of structural part in water and air.

Compared to experiment the geometry was in a way simplified. Structural part was only box with no holes for hanging ropes, no screw holes for sensor mountings, no chamfer on edges and the model was missing all parts of sensors, cabling etc. Fluid part was a cylinder surrounding the mentioned structural part and dimensions were chosen as height $1.5 \mathrm{~m}$ and diameter $0.8 \mathrm{~m}$. Choice of this size is motivated by having domain big enough so it acts like

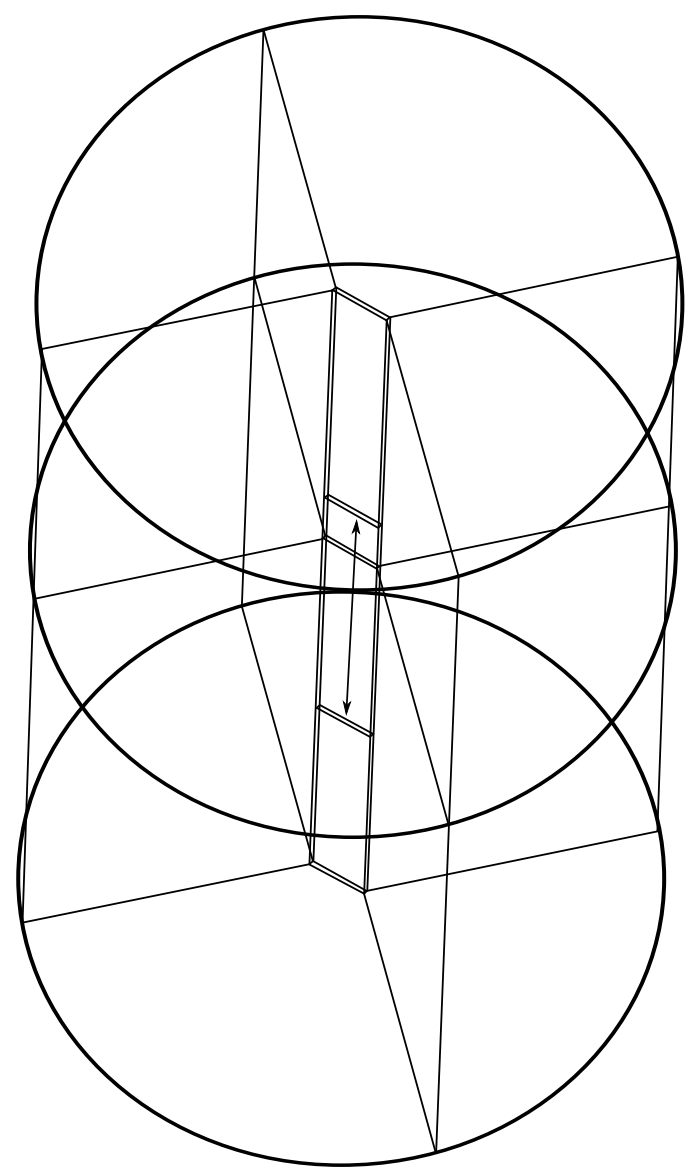

Fig. 4. FEM analysis computational domain (line with arrow ends indicates the plate submersion movement during consecutive simulations. Position of the plate in the picture is $440 \mathrm{~mm}$ submersion.
Table 2. Simulation parameter settings

\begin{tabular}{|c|c|c|}
\hline Parameter & Symbol [Unit] & Value \\
\hline density (water) & $\rho_{w}\left[\mathrm{~kg} \cdot \mathrm{m}^{-3}\right]$ & 1000 \\
\hline density (air) & $\rho_{a}\left[\mathrm{~kg} \cdot \mathrm{m}^{-3}\right]$ & 1.204 \\
\hline density (steel) & $\rho_{s}\left[\mathrm{~kg} \cdot \mathrm{m}^{-3}\right]$ & 7850 \\
\hline sound speed (water) & $c_{w}\left[\mathrm{~m} \cdot \mathrm{s}^{-1}\right]$ & 1500 \\
\hline sound speed (air) & $c_{a}\left[\mathrm{~m} \cdot \mathrm{s}^{-1}\right]$ & 343 \\
\hline Young m. (steel) & $E[\mathrm{~Pa}]$ & $208 \cdot 10^{9}$ \\
\hline Poisson ratio & $\mu[1]$ & 0.3 \\
\hline viscosity (water) & $v_{w}[\mathrm{~Pa} \cdot \mathrm{s}]$ & $1 \cdot 10^{-3}$ \\
\hline viscosity (air) & $v_{a}[\mathrm{~Pa} \cdot \mathrm{s}]$ & $1 \cdot 10^{-5}$ \\
\hline bulk viscosity (w.) & $v_{w(b)}[\mathrm{Pa} \cdot \mathrm{s}]$ & $1 \cdot 10^{3}$ \\
\hline bulk viscosity (a.) & $v_{a(b)}[\mathrm{Pa} \cdot \mathrm{s}]$ & $1 \cdot 10^{2}$ \\
\hline
\end{tabular}

infinite space so only natural frequencies of structural part are gained. This cylinder was split $850 \mathrm{~mm}$ in direction of axis into two parts, the bigger cylindric part was used as acoustic body of water and a smaller one served as an air medium (Fig. 4). Structural part was placed using dimension parameter that was changed during computation to gradualy submerge the plate into a water without a need manualy edit this value after every simulation step.

Parameters used in simulation are written in Tab. 2. Damping properties were used only for a fluid part only and the damping was used to limit and identify specific modes. Mesh size of a ten thousand quadratic HEX20 elements was used and it was sufficent count to provide accurate results and further mesh refinement did not change frequency or stability values. Recommended number of elements [7] (6 quadratic elements in acoustic domain) was taken in account. Boundary condition of a rigid wall was on a three outer faces of cylinder and FSI interface (fluid structure interaction) was on contact surfaces between steel and water (air).

Model with structural part only was used to estimate Young modulus of the measured steel plate since the value of first natural frequency was known from experiment.

There is number of means to identify whether the mode is bending or torsional without a look on a deformed body. Since the damping parameters of water and air were present the value of mode stability number could be used to track particular mode during frequency change caused by submersion in simulation steps. Another way is monitor directional deformation in certain location, using an either corner of a plate results in a bigger deformation in case of twisting modes compared to bending ones. These provided a useful approach to track modes in a data so 


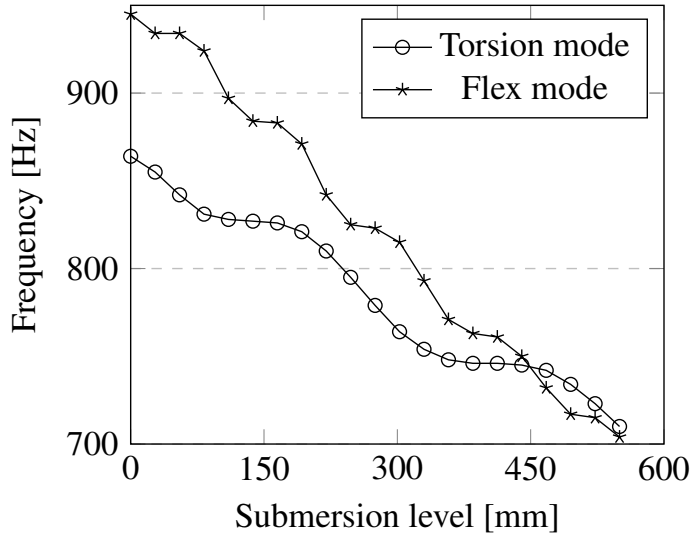

Fig. 5. Second torsion and fourth bending mode during immersion, simulation data

for example the previously mentioned change of order of modes could be easily obtained without visual check on each simulation result. This method was checked on a smaller frequency resolution and then used in further simulations.

In Fig. 5 is visible same situation with type of mode order change this time with computational data. The change between modeshapes happens at a big higher submersion level compared to graph from experiment. Simulation allowed smaller steps of submersion so on the flex mode trend there are visible parts when the five nodes and six anti-nodes were being submerged.

\section{Custom code}

Analytical solution using TMM (transfer matrix method) was created to compare with experimental and computational data. The solution is based on equation describing bending of a steel plate. Gradual change of density was used to include added mass efect of partial immersion. The details of this approach are described in this section. Similar approach to natural frequencies of rotor is solved in [8].

Differential equation of bending of a plate can be written in following form.

$$
\frac{\partial^{2} w}{\partial t^{2}}+c^{2} \cdot \frac{I}{A} \cdot \frac{\partial^{4} w}{\partial x^{4}}=0
$$

Next there is need for a state vector and a relations between its elements.

$$
\begin{gathered}
\mathbf{u}^{T}=\left(\begin{array}{llll}
w & \alpha & M & Q
\end{array}\right) \\
\frac{\partial w}{\partial x}=\alpha \\
M=-E \cdot I \cdot \frac{\partial \alpha}{\partial x} \\
Q=\frac{\partial M}{\partial x}
\end{gathered}
$$

With these equations and few modifications formula for banding can be written using transfer matrix.

$$
\left(\begin{array}{l}
w \\
\alpha \\
M \\
Q
\end{array}\right)_{i+1}=\mathbf{P}_{T}\left(\begin{array}{l}
w \\
\alpha \\
Q
\end{array}\right)_{i}
$$

The transfer matrix is written in next equation.

$$
\mathbf{P}_{T}=\left(\begin{array}{cccc}
S_{p}(\beta x) & \frac{C_{p}(\beta x)}{\beta} & \frac{S_{n}(\beta x)}{\beta^{2} E I} & \frac{S_{n}(\beta x)}{\beta^{3} E I} \\
C_{n}(\beta x) \beta & S_{p}(\beta x) & \frac{C_{n}(\beta x)}{\beta E I} & \frac{S_{N}(\beta x)}{\beta^{2} E I} \\
S_{n}(\beta x) \beta^{2} E I & C_{n}(\beta x) \beta E I & S_{p}(\beta x) & \frac{C_{p}(\beta x)}{\beta} \\
C_{p}(\beta x) \beta^{3} E I & S_{n}(\beta x) \beta^{2} E I & C_{n}(\beta x) \beta & S_{p}(\beta x)
\end{array}\right)
$$

Elements $S_{p}$ and $C_{p}$ are Rayleigh-Krylov functions. In case of $S_{n}$ and $C_{n}$ the sign between hyperbolic and simple goniometric function is negative.

$$
\begin{gathered}
S_{p}(\beta x)=\frac{1}{2}(\sinh \beta x+\sin \beta x) \\
C_{p}(\beta x)=\frac{1}{2}(\cosh \beta x+\cos \beta x)
\end{gathered}
$$

Parameter $\beta$ contains frequency element $\omega$.

$$
\beta^{4}=\frac{\omega^{2} \cdot A}{c^{2} \cdot I}
$$

Speed of sound is a function of Young's modulus and material density.

$$
c^{2}=\frac{E}{\rho}
$$

Table 3. Simulation parameter settings

\begin{tabular}{l|l|l} 
Parameter & Symbol [Unit] & Value \\
\hline $\begin{array}{l}\text { density (steel) } \\
\text { density of submerged } \\
\text { part }\end{array}$ & $\rho_{s}\left[\mathrm{~kg} \cdot \mathrm{m}^{-3}\right]$ & 7850 \\
$\begin{array}{l}\text { Young modulus } \\
\text { Area }\end{array}$ & $E[\mathrm{~Pa}]$ & 15800 \\
Second area moment & $I\left[\mathrm{~m}^{4}\right]$ & $4.8 \cdot 10^{-4}$ \\
\end{tabular}

Solution of eigen value was obtained by finding an extreme value in an assumed frequency span. 
Parameters for computation were same as in FEM ANSYS simulation but not all of them are aplicable, geometry is limited to structural part only and material properties of steel are used. But to substitute effect of submersion, plate is split in two halfs accordingly to immersion level and the submerged part has a different density. This value was determined by eigenfrequency of fully submerged plate so the density of whole plate is the unkonwn value. During following computations this value was constant and only change was a length ratio between two parts with different densities (Tab. 3).

The reason for this approach is that when the structure vibrates the surrounding media vibrates as well. So the increase of density substitues the effect of water around steel plate.

\section{Results}

Comparison of three different methods used to find second natural frequency is presented in Fig. 6. The trends are same for all of them, with evident quick frequency decrease in the first, middle and final part of submersion according to the location of anti-nodes. But there is also visible disagreement, none of the data sets fits with other precisely throught the whole interval. The analytic and

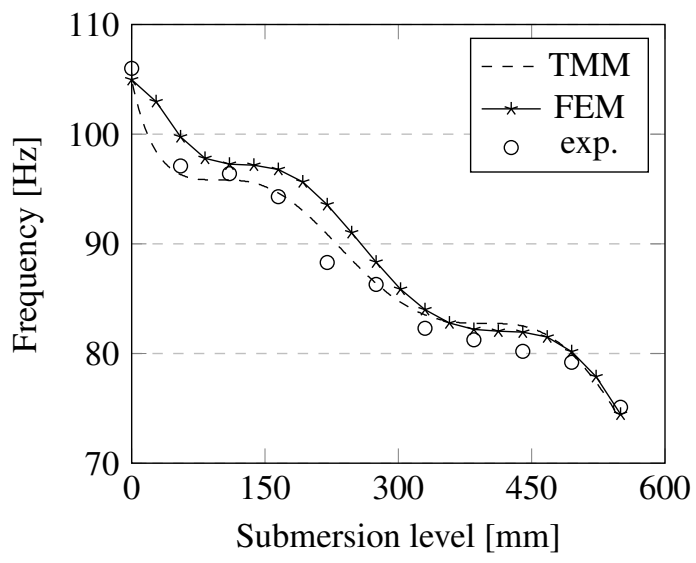

Fig. 6. First natural frequency during immersion

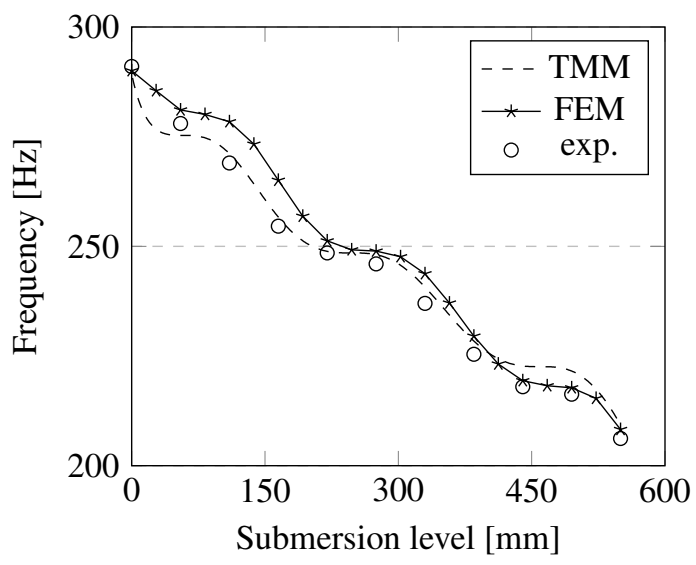

Fig. 7. Second natural frequency during immersion

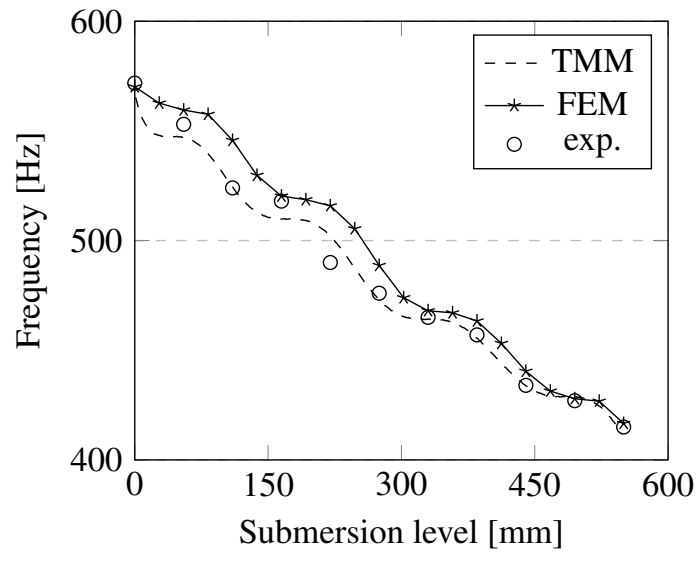

Fig. 8. Third natural frequency during immersion

FEM approach are in agreement in the last part of submersion but not so well during the initial part. It seems that both computational approaches have a different limitations compared to each other and also are missing some physical phenomena that is contained in experimental measurement.

In Fig. 7 and in Fig. 8 the second and third bending modes (second and fourth mode overall, third one is torsional) frequencies of plate in relation to immersion are presented. The trend resembles situation of a first natural frequency, both full immersion and non-submerged condition results in accurate and approximately same values of frequency. Trends show similar course but more or less differ in its values along the way.

Added mass effects in this contribution are reduced to an added density as is described in Custom code section. Since the added density is constant for whole immersed part it would be possible to calculate single value of added mass. This is not as useful as in situation of harmonic vibration of body in one direction for which case the added mass is easily related to simple volume of liquid that vibrates with structure. In presented cases the volume of fluid around the body that vibrates is more complicated because it is deterimed by a mode shape of a particular natural frequency.

\section{Conclusion}

In this paper the influence of immersion on dynamic properties namely natural frequencies of steel plate are presented. To compare data with experimentally obtained values FEM simulation and TMM code is used. The natural frequencies for a fully submerged steel plate and a steel plate without influence of surrounding water are pretty much precise but between these points the sets differ.

Change of order of fifth and sixth modeshape is captured in experiment and in FEM simulation. The change happens in a bit different level of submersion but that can be in part explained by experiment resolution with eleven steps of immersion.

Comparison of first three bending modes shows a difference between used methods and experiment. The transfer matrix based method seems to be more precise then 
FEM simulation in a middle part of submersion interval. This is despite of a simplified approach to added mass effect using constant added density for submerged part of steel plate. Plausible explanation of disagreement with experiment are physical influences included in experiment but ommited in FEM simulation and analytically based TMM computation. Despite these differences this methods offer useful description of behavior of frequency of the steel plate during gradual immersion.

The presented work was performed with support of TA ČR project TE02000232 Special Rotary Machines Engineering Centre.

\section{Nomenclature}

$\begin{array}{lll}A & {\left[\mathrm{~m}^{2}\right]} & \text { area } \\ b & {[\mathrm{~m}],[\mathrm{mm}]} & \text { width of the plate } \\ c & {\left[\mathrm{~m} \mathrm{~s}^{-1}\right]} & \text { speed of sound } \\ E & {[\mathrm{~Pa}]} & \text { Young modulus } \\ I & {\left[\mathrm{~m}^{4}\right]} & \text { second moment of area } \\ L & {[\mathrm{~m}],[\mathrm{mm}]} & \text { length of the plate } \\ M & {[\mathrm{Nm}]} & \text { torque } \\ \mathbf{P} & & \text { transfer matrix } \\ Q & {[\mathrm{~N}]} & \text { Shear force } \\ t & {[\mathrm{~m}],[\mathrm{mm}]} & \text { thickness of the plate } \\ \mathbf{u} & & \text { state vector } \\ w & {[\mathrm{~m}]} & \text { deflection, deformation } \\ x & {[\mathrm{~m}]} & \text { dimension coordinate } \\ \alpha & {[\mathrm{rad}]} & \text { angle of inclination } \\ v & {[\mathrm{~Pa} \mathrm{~s}]} & \text { viscosity } \\ \omega & {\left[\mathrm{rad} \mathrm{s}{ }^{-1}\right]} & \text { eigen value, angular frequency }\end{array}$

\section{References}

1. KRAMER, M. R., Z. LIU, Y. L. YOUNG. Free vibration of cantilevered composite plates in air and in water. Composite Structures. 2013, 95, 254-263. DOI: 10.1016/j.compstruct.2012.07.017 . ISSN 02638223.

2. ERGIN, A., B. UĞURLU. Linear vibration analysis of cantilever plates partially submerged in fluid. Journal of Fluids and Structures. 2003, 17(7), 927-939. DOI: 10.1016/S0889-9746(03)00050-1. ISSN 08899746.

3. DE LA TORRE, O., X. ESCALER, E. EGUSQUIZA, M. FARHAT. Numerical and experimental study of a nearby solid boundary and partial submergence effects on hydrofoil added mass. 2014, 91, 1-9. DOI: 10.1016/j.compfluid.2013.12.003. ISSN 00457930.

4. DA LOZZO, E., F. AURICCHIO, G. CALVI, Added Mass Model for Vertical Circular Cylinder Immersed in Water, Proceedings of the 15th World Conference on Earthquake Engineering, 2012, Lisbon, Portugal, pp. $1-10$.

5. MURRAY, R. E., R. THRESHER, J. JONKMAN. Added mass effects on a horizontal axis tidal turbine using FAST v8. Renewable Energy. 2018, 126, 987-1002. DOI: 10.1016/j.renene.2018.04.023. ISSN 09601481.

6. GREŠÁKOVÁ, K.. Experimental determination of the liquid influence on an oscillating body. Brno University of Technology, 2018 hdl.handle.net/11012/82308. Diploma thesis.

7. ANSYS Inc., Introduction to acoustics (product manual), ANSYS Acoustics Lectures, 2016

8. AMS, A., W. KLEIN. VIB - Verified Inclusions of Critical Bending Vibrations. Scientific computation with automatic result verification. 1988, New York: Springer-Verlag, p. 91-98. Computing (SpringerVerlag), 6. DOI: 10.1007/978-3-7091-6957-5. ISBN 0387820639. 